\title{
Clinical significance of pretreatment prognostic nutritional index and lymphocyte-to-monocyte ratio in patients with advanced p16-negative oropharyngeal cancer - a retrospective study
}

\author{
Ming-Hsien Tsai ${ }^{1,2,}$, Tai-Lin Huang ${ }^{2,4}$, Hui-Ching Chuang ${ }^{1,2}$, Yu-Tsai Lin ${ }^{1,2,3}$, Fu-Min Fang ${ }^{2,5}$, Hui Lu ${ }^{1}$, Chih-Yen \\ Chien ${ }^{\text {Corresp. 1, 2, } 6}$ \\ ${ }^{1}$ Department of Otolaryngology, Kaohsiung Chang Gung Memorial Hospital and Chang Gung University College of Medicine, Kaohsiung, Taiwan \\ 2 Kaohsiung Chang Gung Head and Neck Oncology Group, Cancer Center, Kaohsiung Chang Gung Memorial Hospital, Kaohsiung, Taiwan \\ 3 College of Pharmacy and Health Care, Tajen University, Pingtung County, Taiwan \\ 4 Department of Hematology and Oncology, Kaohsiung Chang Gung Memorial Hospital and Chang Gung University College of Medicine, Kaohsiung, Taiwan \\ 5 Department of Radiation Oncology, Kaohsiung Chang Gung Memorial Hospital, Chang Gung University College of Medicine, Kaohsiung, Taiwan \\ 6 Institute For Translational Research In Biomedicine, Kaohsiung Chang Gung Memorial Hospital, Kaohsiung, Taiwan \\ Corresponding Author: Chih-Yen Chien \\ Email address: cychien3965@adm.cgmh.org.tw
}

Background. Systemic inflammation and nutritional status both play roles in the survival of cancer patients. Therefore, it is important to understand the effects of prognostic nutritional index (PNI) and lymphocyte-to-monocyte ratio (LMR) on the survival of patients with advanced p16-negative oropharyngeal cancer. Methods. A total of 142 patients diagnosed with advanced p16-negative oropharyngeal cancer between 2008 and 2015 were enrolled in this study. All patients received primary treatment with definite concurrent chemoradiotherapy (CCRT). Optimal cutoff values for PNI and LMR were determined using receiver operating characteristic curves for survival prediction. Survival rates for different level of PNI and LMR were estimated and compared using Kaplan-Meier method and log-rank test to see if there were significant effects on these end points, including five-year overall survival (OS), disease-specific survival (DSS), and disease-free survival (DFS) rates. The effects of PNI and LMR on survival were assessed using Cox regression model adjusted for other prognostic factors. Results. The results showed the optimal cutoff values for PNI and LMR were 50.5 and 4.45 , respectively. A high PNI ( $\geqq 50.5$ ) was significantly improved the 5 -year OS. A low LMR (<4.45) was significantly associated with a poor 5-year DFS, DSS, and OS. In multivariate analysis, both PNI and LMR were independent prognosticators for 5-year OS. Conclusions. Elevated pretreatment PNI and LMR are both favorable prognosticators in advanced p16-negative oropharyngeal cancer patients undergoing CCRT. 
1 Clinical significance of pretreatment prognostic nutritional

2 index and lymphocyte-to-monocyte ratio in patients with

3 advanced p16-negative oropharyngeal cancer - a

4 retrospective study

5 Ming-Hsien Tsai ${ }^{1,2,3}$, Tai-Lin Huang ${ }^{2,4}$, Hui-Ching Chuang ${ }^{1,2}$, Yu-Tsai Lin ${ }^{1,2,3}$, Fu-Min Fang ${ }^{2,5}$,

6 Hui Lu ${ }^{1}$, Chih-Yen Chien ${ }^{1,2,6, *}$

7

$8 \quad{ }^{1}$ Department of Otolaryngology, Kaohsiung Chang Gung Memorial Hospital and Chang Gung

9 University College of Medicine, Kaohsiung, Taiwan

$10{ }^{2}$ Kaohsiung Chang Gung Head and Neck Oncology Group, Cancer Center, Kaohsiung Chang Gung

11 Memorial Hospital, Kaohsiung, Taiwan

$12{ }^{3}$ College of Pharmacy and Health Care, Tajen University, Pingtung County, Taiwan

$13{ }^{4}$ Department of Hematology and Oncology, Kaohsiung Chang Gung Memorial

14 Hospital and Chang Gung University College of Medicine, Kaohsiung, Taiwan

$15{ }^{5}$ Department of Radiation Oncology, Kaohsiung Chang Gung Memorial Hospital, Chang Gung

16 University College of Medicine, Kaohsiung, Taiwan

$17{ }^{6}$ Institute for Translational Research in Biomedicine, Kaohsiung Chang Gung Memorial

18 Hospital, Kaohsiung, Taiwan

19 
$20 *$ Corresponding author:

21 Chih-Yen Chien

22 Department of Otolaryngology, Kaohsiung Chang Gung Memorial Hospital

23 Tel: +886-7-7317123 ext. 2533; Fax: +886-7-7313855

24 E-mail address: cychien3965@adm.cgmh.org.tw 


\section{Abstract}

26

\section{Background.}

28 Systemic inflammation and nutritional status both play roles in the survival of cancer patients.

29 Therefore, it is important to understand the effects of prognostic nutritional index (PNI) and

30 lymphocyte-to-monocyte ratio (LMR) on the survival of patients with advanced p16-negative

31 oropharyngeal cancer.

32 Methods.

33 A total of 142 patients diagnosed with advanced p16-negative oropharyngeal cancer between

342008 and 2015 were enrolled in this study. All patients received primary treatment with definite

35 concurrent chemoradiotherapy (CCRT). Optimal cutoff values for PNI and LMR were

36 determined using receiver operating characteristic curves for survival prediction. Survival rates

37 for different level of PNI and LMR were estimated and compared using Kaplan-Meier method

38 and log-rank test to see if there were significant effects on these end points, including five-year

39 overall survival (OS), disease-specific survival (DSS), and disease-free survival (DFS) rates. The

40 effects of PNI and LMR on survival were assessed using Cox regression model adjusted for

41 other prognostic factors.

42 Results. 
43 The results showed the optimal cutoff values for PNI and LMR were 50.5 and 4.45, respectively.

44 A high PNI $(\geqq 50.5)$ was significantly improved the 5-year OS. A low LMR $(<4.45)$ was

45 significantly associated with a poor 5-year DFS, DSS, and OS. In multivariate analysis, both PNI

46 and LMR were independent prognosticators for 5-year OS.

\section{Conclusions.}

48 Elevated pretreatment PNI and LMR are both favorable prognosticators in advanced p16-

49 negative oropharyngeal cancer patients undergoing CCRT. 


\section{Introduction}

51 It is estimated that head and neck cancers are the sixth most commonly diagnosed systemic

52 malignant tumors with more than 500,000 new cases and 300,000 associated deaths annually.

53 (McGuire, 2016) Oropharyngeal squamous cell carcinoma (OPSCC) is an aggressive type of

54 head and neck cancer. The average annual percentage increase of OPSCC was $6.1 \%$ between

551980 and 2014, and the trend continues to increase with numerous OPSCC cases diagnosed as

56 advanced stages in Taiwan. (Hsu et al., 2017) Though investigations of OPSCC have been

57 carried out for decades worldwide, its etiologic and clinical characteristics differ substantially

58 among populations. For instance, human papillomavirus (HPV) infection may lead to a more

59 favorable prognosis in patients than that without HPV infection. (Mehanna et al., 2013) Besides,

60 the relatively low HPV infection rate, approximately $20-30 \%$, in Taiwan along with the high

61 prevalence of betel nut chewing both may deteriorate the prognosis, as investigated in our

62 previous study (Al-Swiahb et al., 2010) makes the evaluation of treatments and possible

63 prognostic factors in advanced stage HPV-negative OPSCC an urgent need.

64 In addition to HPV status, inflammatory biomarkers are thought to be a representation of the

65 interaction between the tumor microenvironment and host immune system.(O'Callaghan et al.,

66 2010; Aggarwal, Vijayalekshmi \& Sung, 2009) Recent studies have shown a negative prognostic

67 value of higher neutrophil-to-lymphocyte ratio and lower lymphocyte-to-monocyte ratio (LMR) 
68 among patients with head and neck cancer.(Perisanidis et al., 2013; Haddad et al., 2015; Rassouli

69 et al., 2015; Tham et al., 2018) Takahashi et al.(Takahashi et al., 2019) reported that a low LMR

70 was an independent adverse prognostic factor for survival in patients with OPSCC.

71 Nutritional impairment has also been shown to have a negative impact on clinical outcomes.

72 (Moon et al., 2016) Patients with advanced stage OPSCC are often vulnerable to malnutrition at

73 the time of diagnosis because of poor food intake due to cancer-related pain, mechanical

74 obstruction by the tumor, or psychological problems. The prognostic nutritional index (PNI),

75 calculated as previously described, (Onodera, Goseki \& Kosaki, 1984) may be especially useful

76 because it could act as a surrogate marker for both inflammation and nutritional status. This

77 index was originally studied to demonstrate the correlation between postoperative complications

78 and prognosis in patients with esophageal carcinoma.(Nozoe et al., 2002) With regard to head

79 and neck cancer, a low PNI had shown as a poor survival predictor in previous study.(Bruixola et

80 al., 2018)

81 Currently, studies on the role of PNI and LMR played in patients with advanced stage HPV-

82 negative OPSCC are still limited. Clinically, p16 expression could be regarded as a surrogate

83 marker for HPV in the prediction of tumor behavior in oropharyngeal cancer. (Golusiński et al.,

84 2017) Thus, the objective of this study was to identify the significant effects of PNI and LMR on

85 clinical prognosis in patients with advanced stage (stage III/IV) p16-negative OPSCC. 


\section{Material and Methods}

\section{Study population.}

88 Patients who were histologically confirmed by biopsy to have stage III/IV p16-negative

89 OPSCC were evaluated in the study. The TNM stage was reclassified according to the 8th

90 edition of the American Joint Committee on Cancer (AJCC) staging system. Patients who were

91 treated with primary concurrent chemoradiotherapy (CCRT) were eligible for this study. The

92 determination of $\mathrm{p} 16$ expression in tumor cells by immunohistochemistry was done as suggested

93 in the 8th edition AJCC staging system manual.(Amin et al., 2017) Patients with clinical

94 evidence of an acute infection within 4 weeks prior to the blood tests or who were diagnosed

95 with recurrent tumors, distant metastases, other concomitant active cancers, or chronic

96 inflammatory disease or who had a history of malignancy in the past 5 years were excluded from

97 the study.

98 In this retrospective study, 142 patients with stage III/IV p16-negative OPSCC who

99 underwent primary CCRT at the Kaohsiung Chang Gung Memorial Hospital in Taiwan between

100 January 2008 and April 2015 were recruited. Treatment was primarily based on the American

101 NCCN guidelines. The chemotherapy agent was cisplatin-based and the radiation technique for

102 all patients was intensity-modulated radiation therapy (IMRT). The primary radiation dose for all

103 of our patients was between 70 and 74 Gy with conventional fractionated daily dose of 1.8 or 2 
104 Gy. All included patients completed the treatment programs formulated by the multidisciplinary

105 team.

106 Variables and outcomes.

107 Pretreatment clinical variables of interest were collected, including age, sex, Adult

108 Comorbidity Evaluation-27 (ACE-27) score, and clinical TNM stage of the tumor. Information

109 collection of pretreatment complete blood count (including absolute lymphocyte and monocyte

110 counts) and biochemical (including albumin) tests using the peripheral blood sample were also

111 conducted within one week before treatment.

112 The LMR was calculated by dividing the baseline absolute peripheral lymphocyte count

$113\left(\right.$ cells $\left./ \mathrm{mm}^{3}\right)$ by the absolute monocyte peripheral count $\left(\right.$ cells $\left./ \mathrm{mm}^{3}\right)$.

114 The PNI was calculated as follows: $10 \times$ baseline serum albumin $(\mathrm{g} / \mathrm{dL})+0.005 \times$ baseline

115 absolute lymphocyte count (cells $\left./ \mathrm{mm}^{3}\right)$.

116 Statistical analysis.

117 The endpoints in our study were the 5-year overall survival (OS), 5-year disease-specific

118 survival (DSS), and 5-year disease-free survival (DFS) rates. Overall survival calculated the time

119 frame from the date of the first treatment to the date of death or last follow-up; disease specific

120 survival calculated from the date of the first treatment to the date of death because of tumor or

121 last follow-up. Disease free survival calculated the time from the date of the first treatment to the 
122 date of recurrence, metastasis, or last follow-up. Follow-up was continued through May 2020.

123 Receiver operating characteristic curves for survival were plotted, and Youden's index, which

124 calculated as $\mathrm{J}=$ sensitivity + specificity -1 , was applied to verify the optimum cutoff value of

125 LMR and PNI for OS. Survival rates of certain prognostic factors were estimated using the

126 Kaplan-Meier method, and the log-rank test was used to determine the heterogeneity of each

127 specific factor. Sex and smoking status variables were excluded from the analysis because of the

128 extremely imbalanced distribution. The Cox proportional hazards model was built with

129 independent primary factors and other significant prognostic factors that were identified in prior

130 univariate survival analyses. The variance inflation factors (VIF) were assessed to avoid

131 multicollinearity among independent variables in the Cox model. Both VIF values for continuous

132 PNI to continuous LMR or dichotomous PNI to dichotomous LMR were below 3 (1.004 and

133 1.002, respectively), which indicated that there was a low correlation between PNI and LMR. All

134 statistics tests were two-sided, with 0.05 significant level. All statistical analyses were performed

135 using the Social Science Software, version 20.0 package (SPSS, Chicago, IL). This study was

136 approved by the Medical Ethics and Human Clinical Trial Committees at Chang Gung Memorial

137 Hospital (Ethical Application Reference number:202000471B0). Patients' consent to review

138 their medical records was not required by this hospital's committees because the patient data

139 remained anonymous in this study. 


\section{Results}

141 Of the 142 p16-negative OPSCC patients, 99.3\% (141) were men and $0.7 \%$ (1) were women.

142 The mean age at diagnosis for all participants was 53.8 years (range: $36-85$ years). The mean

143 follow-up period was 40.7 months (3.6-111.8 months). Nine patients (6.3\%) had stage III

144 disease, 34 (23.9\%) had stage IVA, and 99 (69.7\%) had stage IVB. This cohort included patients

145 with clinical T classifications of T1 $(n=4,2.8 \%)$, T2 $(n=24,16.9 \%)$, T3 $(n=24,16.9 \%)$, T4a

$146(\mathrm{n}=34,23.9 \%)$, and $\mathrm{T} 4 \mathrm{~b}(\mathrm{n}=56,39.4 \%)$. Clinical nodal metastasis was present in 117 patients

147 (82.4\%), and 65 (45.8\%) had extranodal extensions (ENE). The clinicopathological features of

148 the 142 cases, and their survival outcomes were listed in Table 1.

149 The optimal cutoff value for PNI was 50.5, and 4.45 for LMR (Figure 1). Patients with

$150 \mathrm{PNI} \geqq 50.5$ or $\mathrm{LMR} \geqq 4.45$ did not have significant correlations with age, $\mathrm{T}$ classification, $\mathrm{N}$

151 classification, or other clinicopathologic factors (all $p>0.05$; Table S1).

152 The OS rate for patients with $\mathrm{PNI} \geqq 50.5$ was significantly higher than that for patients with

$153 \mathrm{PNI}<50.5$ (48.1\% vs $24.7 \%, p=0.004)$. Similarly, the DSS for patients with PNI $\geqq 50.5$ was

154 significantly higher than that for patients with $\mathrm{PNI}<50.5(57.2 \%$ vs $42 \%, p=0.043)$. Moreover,

155 DFS had a similar trend by PNI difference in our cohort (44.3\% vs 34.2\%), although $p$ value did

156 not reach statistical significance $(p=0.108$, Figure 2$)$. Regarding the LMR, the 5-year OS $(55.5 \%$

157 vs $26.6 \%)$, DSS (66.8\% vs $41.4 \%)$ and DFS (51.4\% vs 35.0\%) were all significantly increased 
158 (both $p<0.05$, Figure 3) among patients with LMR $\geqq 4.45$, compared with those with LMR $<4.45$.

159 Clinically positive ENE status was another significant predictor of poor outcome for 5-year OS,

160 DSS, and DFS in univariate analysis (Table 2).

161 Multiple regression analysis was applied to analyze the relationship between survival

162 outcome and significant factors which were revealed in prior univariate analyses. PNI was an

163 independent factor of OS in this cohort (hazard ratio [HR]: $1.778,95 \%$ confidence interval [CI]:

$1641.145-2.761$ ) and simultaneously adjusted by other independent factors, LMR and ENE (Table

165 3). In another model, the status of LMR showed a significant prognosticator in OS (HR of 2.408,

166 95\% CI: 1.439-4.029), DSS (HR: 2.33, 95\% CI: 1.255-4.323), and DFS (HR: $1.765,95 \%$ CI:

167 1.067-2.892) after being adjusted by other factors (Tables 3-5). The status of clinical ENE was a

168 significant prognosticator of OS (HR: 1.592, 95\% CI: 1.054-2.405), DSS (HR: 2.159, 95\% CI:

169 1.319-3.533), and DFS (HR: 1.86, 95\% CI: 1.202-2.878). (Tables 3-5) 


\section{Discussion}

171 In the current study of patients with advanced stage (stage III/IV) p16-negative OPSCC, the

172 5-year DFS, DSS, and OS rates were 39.9\%, 49.8\%, and 35.6\%, respectively. PNI, LMR, and

173 clinical ENE status were all significant independent factors of OS in our multivariate cox

174 regression analysis.

175 Clinical ENE is the extension of metastatic lymph node through an affected lymph node

176 capsule. It has always been considered a marker of poor prognosis as tumor recurrence and

177 oncological survival in head and neck cancer; thus, it was proposed to be incorporated into the

178 newest edition of the AJCC staging system manual (Amin et al., 2017). Our cohort also revealed

179 similar results, showing that the presence of ENE was associated with poor oncologic outcomes.

180 A low PNI indicated a decrease in the serum albumin and/or a low absolute lymphocyte

181 count. Serum albumin is an important factor of the host inflammatory response and nutritional

182 status.(Gupta \& Lis, 2010) The absolute lymphocyte count is also believed to be an important

183 participant in the inhibition of cancer growth by initiating a cytotoxic immune

184 response.(Mantovani et al., 2008) Taken together, this existing evidence showed that

185 malnutrition and lymphocytopenia may be factors affecting a chronically impaired immune

186 system. The cutoff value for PNI reported in previous studies in other type of cancer was $40-$

187 60.(Feng \& Chen, 2014; Lee et al., 2017; Jian-Hui et al., 2016; Shibutani et al., 2015; Yang et 
188 al., 2016; Zhang et al., 2018) With regard to head and neck cancer, several studies found that

189 lower PNI predicted poor oncologic outcomes in head and neck squamous cell carcinoma

190 (HNSCC) (Table 6).(Bruixola et al., 2018; Kono et al., 2017; Chang et al., 2018; Fu et al., 2016)

191 Bruixola et al. demonstrated that low PNI (cutoff value: 45) was an independent prognostic

192 biomarker in locoregional advanced HNSCC.(Bruixola et al., 2018) Fu et al. studied 975 patients

193 with laryngeal squamous cell carcinoma treated with curative laryngectomy, and found that

194 patients with PNI $<48.65$ had a low probability of cancer-specific survival and OS.(Fu et al.,

195 2016) Our results are comparable with these findings, showing that a low PNI is an indicator of

196 poor prognosis in patients with advanced stage (stage III/IV) p16-negative OPSCC undergoing

197 primary CCRT, with a cutoff value similar to previous studies.(Bruixola et al., 2018; Kono et al.,

198 2017; Chang et al., 2018; Fu et al., 2016) In our study, patients with PNI < 50.5 had significantly

199 reduced survival with adjusted for other prognostic factors in the multivariate analysis.

200 Studies of investigating the clinical effects of LMR on HNSCC prognosis have increased in

201 recent years. White blood cell differential could be divided into myeloid lineage and lymphoid

202 lineage. It is believed that lymphoid lineage preponderance of white blood cell was related to

203 better survival based on previous HNSCC study. (Wu et al., 2017) Several studies found that

204 lower LMR predicted reduced DSS and OS in HNSCC (Table 7).(Takahashi et al., 2019; Tham

205 et al., 2019; Furukawa et al., 2019; Yang et al., 2018; Kano et al., 2017) In addition, the 
206 relationship between LMR and advanced stage OPSCC was not thoroughly evaluated. Our

207 results are comparable with these findings, showing that a low LMR is an indicator of poor

208 prognosis in advanced stage (stage III/IV) p16-negative OPSCC. Patients with LMR $<4.45$ have

209 significantly reduced OS, DSS, and DFS according to the multivariate analysis.

210 The mechanism between an increased systemic inflammatory response and promotion of

211 tumor cell invasion is not clearly understood. A possible explanation might lie in the antitumoral

212 roles that lymphocyte plays by inhibiting tumor cell proliferation and migration, and reinforcing

213 human's immune response to cancer. (De Giorgi et al., 2012) Fewer infiltrating lymphocytes

214 have been correlated to poor prognosis.(Gooden et al., 2011) In contrast, higher levels of

215 monocyte-derived macrophages have been associated with greater tumor aggressiveness and

216 poorer survival outcomes.(Pollard et al., 2004) This is postulated to happen through tumor

217 microenvironment mediators such as TNF- $\alpha$, vascular endothelial growth factor, and epidermal

218 growth factor.(Pollard et al., 2004; Xiong et al., 1998) A low LMR implies a relative decrease in

219 lymphocytes and / or increase monocytes. Perhaps, the prognostic ability of LMR is owing to its

220 action as a crude marker for the pro-tumor versus anti-tumor dynamic in the immune

221 system.(Lin, Chien \& Chuang, 2017)

222 PNI, which calculated as $10 \times$ baseline serum albumin $(\mathrm{g} / \mathrm{dL})+0.005 \times$ baseline absolute

223 lymphocyte count $\left(\right.$ cells $\left./ \mathrm{mm}^{3}\right)$, is used to evaluate the immune-nutritional status and may 
224 influence the prognosis of cancer patients.(Yao et al., 2013) Poor immune-nutritional status has

225 been reported as its' association with an immunosuppressed condition, which provides a

226 favorable microenvironment for tumor relapse.(Colotta et al., 2009) That may be the reason why

227 this immunosuppressed condition in low-PNI patients may cause the poor outcomes. Recently,

228 remarkable progress in research on immune checkpoints in tumor immunity has allowed the

229 elucidation of the molecular mechanism underlying immunological tolerance to tumor

230 development. The association between peripheral inflammatory biomarkers and treatment

231 outcomes for immunotherapy remains unclear. These biomarkers might serve as a useful

232 predictor for immunotherapy in the treatment of head and neck cancer in the future.

233 In our study, we have identified the clinical significance of PNI and LMR on survival in

234 patients with p16 negative oropharyngeal cancer treated by CCRT. Moreover, we had control

235 cancer stage and HPV status these two well-known prognostic factors in oropharyngeal cancer,

236 making this cohort homogenous for our analysis findings. However, the drawback of our study is

237 that it is retrospective, and selective bias may exist. A prospective study or large series study

238 from multiple institutes is necessary to confirm our findings. 


\section{Conclusion}

241 In summary, our current study showed that patients with higher pretreatment LMR $(\geqq 4.45)$

242 showed significantly better survival than those with lower $\operatorname{LMR}(<4.45)$; Patients with higher

243 PNI ( $\geqq 50.5$ ) revealed significantly better 5-year OS and 5-year DSS than those with lower PNI

$244(<50.5)$. According to Cox regression analysis from this cohort, pretreatment LMR and PNI

245 were also an independent prognostic factor that predicts OS. Interestingly, it may be possible to

246 incorporate pretreatment LMR and PNI into the treatment strategy for patients with advanced

247 stage p16-negative OPSCC undergoing CRT/RTO in the future. 


\section{Reference}

249 1. McGuire S. 2016. World Cancer Report 2014. Geneva, Switzerland: World Health

250 Organization, International Agency for Research on Cancer, WHO Press, 2015.

251

Advances in nutrition, 7: 418-419. DOI:10.3945/an.116.012211

252 2. Hsu WL, Yu KJ, Chiang CJ, Chen TC, Wang CP. 2017. Head and Neck Cancer

253 Incidence Trends in Taiwan, $1980 \sim 2014$. International Journal of Head and Neck

Science, 1:180-189. DOI: 10.6696/IJHNS.2017.0103.05

3. Mehanna H, Beech T, Nicholson T, El-Hariry I, McConkey C, Paleri V, Roberts S. 2013.

Prevalence of human papillomavirus in oropharyngeal and nonoropharyngeal head and neck cancer--systematic review and meta-analysis of trends by time and region. Head \&

neck, 35:747-755. DOI: 10.1002/hed.22015

4. Al-Swiahb JN, Huang CC, Fang FM, Chuang HC, Huang HY, Luo SD, Chen CH, Chen CM, Chien CY. 2010. Prognostic impact of p16, p53, epidermal growth factor receptor, and human papillomavirus in oropharyngeal cancer in a betel nut-chewing area. Archives

5. O'Callaghan DS, O'Donnell D, O'Connell F, O'Byrne KJ. 2010. The role of inflammation in the pathogenesis of non-small cell lung cancer. Journal of thoracic oncology, 5:2024- 
266 6. Aggarwal BB, Vijayalekshmi RV, Sung B. 2009. Targeting inflammatory pathways for prevention and therapy of cancer: short-term friend, long-term foe. Clinical cancer research, 15:425-430. DOI: 10.1158/1078-0432.CCR-08-0149

7. Perisanidis C, Kornek G, Pöschl PW, Holzinger D, Pirklbauer K, Schopper C, Ewers R. 2013. High neutrophil-to-lymphocyte ratio is an independent marker of poor diseasespecific survival in patients with oral cancer. Medical oncology, 30:334. DOI: $10.1007 / \mathrm{s} 12032-012-0334-5$

8. Haddad CR, Guo L, Clarke S, Guminski A, Back M, Eade T. 2015. Neutrophil-tolymphocyte ratio in head and neck cancer. Journal of medical imaging and radiation oncology, 59:514-519. DOI: 10.1111/1754-9485.12305

9. Rassouli A, Saliba J, Castano R, Hier M, Zeitouni AG. 2015. Systemic inflammatory markers as independent prognosticators of head and neck squamous cell carcinoma. Head

10. Tham T, Olson C, Khaymovich J, Herman SW, Costantino PD. 2018. The lymphocyteto-monocyte ratio as a prognostic indicator in head and neck cancer: a systematic review and meta-analysis. European archives of oto-rhino-laryngology, 275:1663-1670. DOI: 
283 11. Takahashi H, Sakakura K, Tada H, Kaira K, Oyama T, Chikamatsu K. 2019. Prognostic

284 significance and population dynamics of peripheral monocytes in patients with

285 oropharyngeal squamous cell carcinoma. Head \& Neck, 41:1880-1888. DOI:

286

10.1002/hed.25625

287

12. Moon H, Roh JL, Lee SW, Kim SB, Choi SH, Nam SY, Kim SY. 2016. Prognostic value

of nutritional and hematologic markers in head and neck squamous cell carcinoma treated

by chemoradiotherapy. Radiotherapy and oncology, 118:330-334. DOI:

10.1016/j.radonc.2015.10.029

291

13. Onodera T, Goseki N, Kosaki G. 1984. Prognostic nutritional index in gastrointestinal surgery of malnourished cancer patients. Nihon Geka Gakkai Zasshi, 85:1001-1005.

14. Nozoe T, Kimura Y, Ishida M, Saeki H, Korenaga D, Sugimachi K. 2002. Correlation of pre-operative nutritional condition with post-operative complications in surgical treatment for oesophageal carcinoma. European journal of surgical oncology, 28:396400. DOI: $10.1053 /$ ejso.2002.1257

15. Bruixola, G., Caballero, J., Papaccio, F., Petrillo, A., Iranzo, A., Civera, M., Moriana, M., 
300 squamous cell head and neck cancer. ESMO Open, 3:e000425. DOI: 10.1136/esmoopen-

$301 \quad 2018-000425$

302 16. Golusiński P, Pazdrowski J, Szewczyk M, Misiołek M, Pietruszewska W, Klatka J, Okła

303 S, Kaźmierczak H, Marszałek A, Filas V, Schneider A, Masternak MM, Stęplewska K,

304 Miśkiewicz-Orczyk K, Golusiński W. 2017. Is immunohistochemical evaluation of p16

305 in oropharyngeal cancer enough to predict the HPV positivity? Reports of Practical

306 Oncology \& Radiotherapy, 22:237-242. DOI: 10.1016/j.rpor.2017.01.003

307 17. Amin MB, Greene FL, Edge SB, Compton CC, Gershenwald JE, Brookland RK, Meyer

308 L, Gress DM, Byrd DR, Winchester DP. 2017. The Eighth Edition AJCC Cancer Staging

309 Manual: Continuing to build a bridge from a population-based to a more "personalized"

310 approach to cancer staging. CA: a cancer journal for clinicians, 67:93-99. DOI:

$311 \quad 10.3322 /$ caac. 21388

312 18. Gupta D, Lis CG. 2010. Pretreatment serum albumin as a predictor of cancer survival: a

313 systematic review of the epidemiological literature. Nutrition journal, 9:69. DOI:

$314 \quad 10.1186 / 1475-2891-9-69$

315 19. Mantovani A, Allavena P, Sica A, Balkwill F. 2008. Cancer-related inflammation. 
317 20. Feng JF, Chen QX. 2014. Significance of the prognostic nutritional index in patients with

318 esophageal squamous cell carcinoma. Therapeutics and clinical risk management, 10:1-7.

319 DOI: $10.2147 /$ TCRM.S56159

320 21. Lee SH, Chung MJ, Kim B, Lee HS, Lee HJ, Heo JY, Kim YJ, Park JY, Bang S, Park

321 SW, Song SY, Chung JB. 2017. The Significance of the Prognostic Nutritional Index for

322 All Stages of Pancreatic Cancer. Nutrition and cancer, 69:512-519. DOI:

323

$10.1080 / 01635581.2016 .1250921$

324 22. Jian-Hui C, Iskandar EA, Cai ShI, Chen CQ, Wu H, Xu JB, He YL. 2016. Significance of

325 Onodera's prognostic nutritional index in patients with colorectal cancer: a large cohort

326 study in a single Chinese institution. Tumour biology, 37:3277-3283. DOI:

$327 \quad 10.1007 / \mathrm{s} 13277-015-4008-8$

328 23. Shibutani1 M, Maeda1 K, Nagahara1 H, Ohtani1 H, Iseki1 Y, Ikeya1 T, Sugano1 K,

Hirakawa1 K. 2015. The prognostic significance of the postoperative prognostic

330 nutritional index in patients with colorectal cancer. BMC Cancer, 15:521. DOI:

331

$10.1186 / \mathrm{s} 12885-015-1537-\mathrm{x}$

332 24. Yang L, Xia L, Wang Y, Hong S, Chen H, Liang S, Peng P, Chen Y. 2016. Low

Prognostic Nutritional Index (PNI) Predicts Unfavorable Distant Metastasis-Free 
334 Survival in Nasopharyngeal Carcinoma: A Propensity Score-Matched Analysis. PLoS

335 One, 11:e0158853. DOI: 10.1371/journal.pone.0158853

336 25. Zhang H, Shang X, Ren P, Gong L, Ahmed A, Ma Z, Ma R, Wu X, Xiao X, Jiang H,

337 Tang P, Yu Z. 2019. The predictive value of a preoperative systemic immune-

338 inflammation index and prognostic nutritional index in patients with esophageal

339 squamous cell carcinoma. Journal of cellular physiology, 234:1794-1802. DOI:

$340 \quad 10.1002 / \mathrm{jcp} .27052$

341 26. Kono T, Sakamoto K, Shinden S, Ogawa K. 2017. Pre-therapeutic nutritional assessment

342 for predicting severe adverse events in patients with head and neck cancer treated by

343 radiotherapy. Clinical nutrition, 36:1681-1685. DOI: 10.1016/j.clnu.2016.10.021

344 27. Chang PH, Hsieh JC, Yeh KY, Chen EY, Yang SW, Huang JS, Lai CH, Wu TH, Huang

345 YM, Chang YS, Chou WC, Wang CH. 2018. Prognostic nutritional index relevance in

346 chemoradiotherapy for advanced oral cavity, oropharyngeal and hypopharyngeal cancer.

347 Asia Pacific journal of clinical nutrition, 27:996-1001. DOI: 10.6133/apjcn.032018.04

348 28. Fu Y, Chen SW, Chen SQ, Ou-Yang D, Liu WW, Song M, Yang AK, Zhang Q. 2016. A

349 Preoperative Nutritional Index for Predicting Cancer-Specific and Overall Survival in

350 Chinese Patients With Laryngeal Cancer: A Retrospective Study. Medicine, 95:e2962. 
352 29. Wu CN, Chuang HC, Lin YT, Fang FM, Li SH, Chien CY. 2017. Prognosis of neutrophil-to-lymphocyte ratio in clinical early-stage tongue (cT1/T2N0) cancer.

30. Tham T, Wotman M, Chung C, Ahn S, Dupuis H, Gliagias V, Movsesova T, Kraus D, Costantino P. 2019. Systemic immune response in squamous cell carcinoma of the head and neck: a comparative concordance index analysis. European archives of oto-rhino-

laryngology, 276:2913-2922. DOI: 10.1007/s00405-019-05554-x

31. Furukawa K, Kawasaki G, Naruse T, Umeda M. 2019. Prognostic Significance of Anticancer research, 39:405-412. DOI: 10.21873/anticanres.13126

32. Yang J, Hsueh CY, Cao W, Zhou L. 2018. Pretreatment lymphocyte-to-monocyte ratio as an independent prognostic factor for hypopharyngeal squamous cell carcinoma. Acta otolaryngologica, 138:734-740. DOI: 10.1080/00016489.2018.1449965 2017. Pretreatment lymphocyte-to-monocyte ratio as an independent prognostic factor for head and neck cancer. Head \& neck, 39:247-253. DOI: 10.1002/hed.24576

34. De Giorgi U, Mego M, Scarpi E, Giuliano M, Giordano A, Reuben JM, Valero V, Ueno 
370 circulating tumor cells as prognostic factors for overall survival in metastatic breast cancer. Clinical breast cancer, 12:264-269. DOI: 10.1016/j.clbc.2012.04.004

372 35. Gooden MJ, de Bock GH, Leffers N, Daemen T, Nijman HW. 2011. The prognostic influence of tumour-infiltrating lymphocytes in cancer: a systematic review with metaanalysis. British journal of cancer, 105:93-103. DOI: 10.1038/bjc.2011.189

375 36. Pollard JW. 2004. Tumour-educated macrophages promote tumour progression and metastasis. Nature reviews. Cancer, 4:71-78. DOI: 10.1038/nrc1256

37. Xiong M, Elson G, Legarda D, Leibovich SJ. 1998. Production of vascular endothelial growth factor by murine macrophages: regulation by hypoxia, lactate, and the inducible nitric oxide synthase pathway. The American journal of pathology, 153:587-598. DOI: $10.1016 / \mathrm{S} 0002-9440(10) 65601-5$

38. Lin CN, Chien CY, Chuang HC. 2017. Are friends or foes? New strategy for head and neck squamous cell carcinoma treatment via immune regulation. International Journal of Head and Neck Science, 1:105-13. DOI: 10.6696/IJHNS.2017.0102.03

384 39. Yao ZH, Tian GY, Wan YY, Kang YM, Guo HS, Liu QH, Lin DJ. 2013. Prognostic nutritional index predicts outcomes of malignant pleural mesothelioma. Journal of cancer research and clinical oncology, 139:2117-2123. DOI: 10.1007/s00432-013-1523-0 
387 40. Colotta F, Allavena P, Sica A, Garlanda C, Mantovani A. 2009. Cancer-related

388 inflammation, the seventh hallmark of cancer: links to genetic instability. Carcinogenesis,

389 30:1073-1081. DOI: 10.1093/carcin/bgp127 


\section{Table $\mathbf{1}$ (on next page)}

Clinical characteristics of 142 patients who were diagnosed with advanced p16-negative OPSCC. 
1 Table 1. Clinical characteristics of 142 patients who were diagnosed with advanced p16-negative OPSCC.

\begin{tabular}{|c|c|c|c|}
\hline \multicolumn{2}{|l|}{ Characteristics } & Value & $\%$ \\
\hline \multicolumn{2}{|l|}{ Mean Age(range), yr } & \multicolumn{2}{|c|}{$53.8(36,85)$} \\
\hline \multicolumn{2}{|c|}{ Mean follow up time (range), months } & \multicolumn{2}{|c|}{$40.7(3.7,111.8)$} \\
\hline \multirow[t]{2}{*}{ Sex } & male & 141 & 99.3 \\
\hline & female & 1 & 0.7 \\
\hline \multirow[t]{3}{*}{ Clinical TNM Stage } & III & 9 & 6.3 \\
\hline & IVA & 34 & 23.9 \\
\hline & IVB & 99 & 69.7 \\
\hline \multirow[t]{5}{*}{ Clinical T classification } & $\mathrm{T} 1$ & 4 & 2.8 \\
\hline & $\mathrm{T} 2$ & 24 & 16.9 \\
\hline & $\mathrm{T} 3$ & 24 & 16.9 \\
\hline & $\mathrm{T} 4 \mathrm{a}$ & 34 & 23.9 \\
\hline & $\mathrm{T} 4 \mathrm{~b}$ & 56 & 39.4 \\
\hline \multirow[t]{5}{*}{ Clinical $\mathrm{N}$ classification } & No & 25 & 17.6 \\
\hline & N1 & 15 & 10.6 \\
\hline & $\mathrm{N} 2 \mathrm{~b}$ & 17 & 12 \\
\hline & $\mathrm{N} 2 \mathrm{c}$ & 20 & 14.1 \\
\hline & $\mathrm{N} 3 \mathrm{~b}$ & 65 & 45.8 \\
\hline \multirow[t]{2}{*}{ Clinical ENE } & negative & 77 & 54.2 \\
\hline & positive & 65 & 45.8 \\
\hline \multirow[t]{3}{*}{ PNI } & unknown & 7 & 4.9 \\
\hline & $<50.5$ & 79 & 55.6 \\
\hline & $\geqq 50.5$ & 56 & 39.4 \\
\hline \multirow[t]{2}{*}{ LMR } & $<4.45$ & 99 & 69.7 \\
\hline & $\geqq 4.45$ & 43 & 30.3 \\
\hline \multirow[t]{2}{*}{ Recurrence } & No & 59 & 41.5 \\
\hline & Yes & 83 & 58.5 \\
\hline \multirow[t]{4}{*}{ Last status } & NED & 35 & 24.6 \\
\hline & Alive with disease & 12 & 8.5 \\
\hline & DOD & 68 & 47.9 \\
\hline & DWOD & 27 & 19.0 \\
\hline
\end{tabular}

\section{3}

3 Abbreviations

40103 
5 Abbreviations

6 Abbreviations: OPSCC, oropharyngeal squamous cell carcinoma; PNI, prognostic nutritional index $=10$ * serum

7 albumin $(\mathrm{g} / \mathrm{dl})+0.005 *$ total lymphocyte count $\left(/ \mathrm{mm}^{3}\right)$; ENE, extranodal extension; LMR, lymphocyte to

8 monocyte ratio; NED, no evidence of disease; DOD, died of disease; DWOD, die without disease. 
Table 2 (on next page)

Univariate Analysis of Factors Impacting Survival $(n=142)$ 
1 Table 2. Univariate Analysis of Factors Impacting Survival $(\mathrm{n}=142)$.

\begin{tabular}{|c|c|c|c|c|c|c|c|c|c|c|c|}
\hline Variable & & Number & Event & $\begin{array}{c}5 \mathrm{yr} \text { OS } \\
(\%)\end{array}$ & $p$ & Event & $\begin{array}{c}5 \text { yr DSS } \\
(\%)\end{array}$ & $p$ & Event & $\begin{array}{c}5 \text { yr DFS } \\
(\%)\end{array}$ & $p$ \\
\hline \multirow{2}{*}{ Age } & $<53$ & 70 & 45 & 38.1 & \multirow{2}{*}{0.489} & 34 & 49.5 & \multirow{2}{*}{0.836} & 44 & 35.3 & \multirow{2}{*}{0.743} \\
\hline & $\geqq 53$ & 72 & 50 & 33.2 & & 34 & 50.1 & & 39 & 44.3 & \\
\hline \multirow{3}{*}{ ACE-27 } & 0 & 93 & 60 & 37.9 & \multirow{3}{*}{0.602} & 44 & 50.4 & \multirow{3}{*}{0.948} & 56 & 39.1 & \multirow{3}{*}{0.893} \\
\hline & 1 & 42 & 30 & 31.4 & & 21 & 47.7 & & 24 & 39.9 & \\
\hline & 2 & 7 & 5 & 28.6 & & 3 & 57.1 & & 3 & 57.1 & \\
\hline Betel nut & no & 36 & 26 & 36.1 & \multirow{2}{*}{0.841} & 16 & 55.7 & \multirow{2}{*}{0.672} & 18 & 48.7 & \multirow{2}{*}{0.416} \\
\hline chewing & yes & 106 & 69 & 35.5 & & 52 & 47.7 & & 65 & 36.9 & \\
\hline Alcohol & no & 24 & 13 & 49.0 & \multirow{2}{*}{0.226} & 9 & 61.6 & \multirow{2}{*}{0.327} & 11 & 52.1 & \multirow{2}{*}{0.221} \\
\hline drinking & yes & 118 & 82 & 32.8 & & 59 & 46.9 & & 72 & 37.3 & \\
\hline Clinical T & $\mathrm{T} 1 / 2 / 3$ & 52 & 34 & 33.5 & \multirow{2}{*}{0.693} & 24 & 50.5 & \multirow{2}{*}{0.638} & 29 & 43.6 & \multirow{2}{*}{0.407} \\
\hline classification & $\mathrm{T} 4 \mathrm{a} / \mathrm{b}$ & 90 & 61 & 36.7 & & 44 & 49.4 & & 54 & 37.7 & \\
\hline Clinical N & N0 & 25 & 18 & 35.6 & \multirow{2}{*}{0.684} & 11 & 50.7 & \multirow{2}{*}{0.801} & 15 & 36.3 & \multirow{2}{*}{0.884} \\
\hline classification & N1-N3b & 117 & 77 & 35.5 & & 57 & 49.6 & & 68 & 40.6 & \\
\hline \multirow{2}{*}{ Clinical ENE } & negative & 77 & 48 & 41.0 & \multirow{2}{*}{$0.037^{*}$} & 29 & 59.4 & \multirow{2}{*}{$0.003 *$} & 38 & 49.7 & \multirow{2}{*}{$0.008^{*}$} \\
\hline & positive & 65 & 47 & 29.1 & & 39 & 38.6 & & 45 & 28.2 & \\
\hline \multirow{2}{*}{ PNI } & $<50.5$ & 79 & 61 & 24.7 & \multirow{2}{*}{$0.004 *$} & 43 & 42.0 & \multirow{2}{*}{$0.043^{*}$} & 50 & 34.2 & \multirow{2}{*}{0.108} \\
\hline & $\geqq 50.5$ & 56 & 31 & 48.1 & & 23 & 57.2 & & 31 & 44.3 & \\
\hline \multirow{2}{*}{ LMR } & $<4.45$ & 99 & 75 & 26.6 & \multirow{2}{*}{$0.001 *$} & 54 & 41.4 & \multirow{2}{*}{$0.01 *$} & 62 & 35.0 & \multirow{2}{*}{$0.042 *$} \\
\hline & $\geqq 4.45$ & 43 & 20 & 55.5 & & 14 & 66.8 & & 21 & 51.4 & \\
\hline
\end{tabular}

2 Abbreviations: *, statistically significant ( $\mathrm{p}<0.05$ ); OS, overall survival; DSS, disease specific survival; DFS,

3 disease free survival; ACE-27: Adult Comorbidity Evaluation-27; ENE, extranodal extension; PNI, prognostic

4 nutritional index; LMR, lymphocyte to monocyte ratio.

5

6 


\section{Table 3 (on next page)}

Multivariate analysis of prognostic factors associated to overall survival. 
1 Table 3. Multivariate analysis of prognostic factors associated to overall survival.

\begin{tabular}{|c|c|c|c|}
\hline Factor & Hazard ratio & $95 \% \mathrm{CI}$ & $p$-value \\
\hline PNI & & & $0.01 *$ \\
\hline$\geqq 50.5$ & 1 & & \\
\hline$<50.5$ & 1.778 & $(1.145,2.761)$ & \\
\hline LMR & & & $0.001 *$ \\
\hline$\geqq 4.45$ & 1 & & \\
\hline$<4.45$ & 2.408 & $(1.439,4.029)$ & \\
\hline ENE & & & $0.027^{*}$ \\
\hline negative & 1 & & \\
\hline positive & 1.592 & $(1.054,2.405)$ & \\
\hline
\end{tabular}

2 Abbreviations: *, statistically significant $(\mathrm{p}<0.05)$; ENE, extranodal

3 extension; PNI, prognostic nutritional index; LMR, lymphocyte to

4 monocyte ratio. 


\section{Table 4 (on next page)}

Multivariate analysis of prognostic factors associated to disease-specific survival. 
1 Table 4. Multivariate analysis of prognostic factors associated to disease-specific survival.

\begin{tabular}{|c|c|c|c|}
\hline Factor & Hazard ratio & $95 \% \mathrm{CI}$ & $p$-value \\
\hline PNI & & & 0.066 \\
\hline$\geqq 50.5$ & 1 & & \\
\hline$<50.5$ & 1.624 & $(0.968,2.723)$ & \\
\hline LMR & & & $0.007^{*}$ \\
\hline$\geqq 4.45$ & 1 & & \\
\hline$<4.45$ & 2.33 & $(1.255,4.323)$ & \\
\hline ENE & & & $0.002 *$ \\
\hline negative & & & \\
\hline positive & 2.159 & $(1.319,3.533)$ & \\
\hline
\end{tabular}

2 Abbreviations: *, statistically significant $(\mathrm{p}<0.05)$; ENE, extranodal

3 extension; PNI, prognostic nutritional index; LMR, lymphocyte to

4 monocyte ratio. 


\section{Table 5 (on next page)}

Multivariate analysis of prognostic factors associated to disease-free survival. 
1 Table 5. Multivariate analysis of prognostic factors associated to disease-free survival.

\begin{tabular}{|c|c|c|c|}
\hline Factor & Hazard ratio & $95 \% \mathrm{CI}$ & $p$-value \\
\hline LMR & & & $0.027^{*}$ \\
\hline$\geqq 4.45$ & 1 & & \\
\hline$<4.45$ & 1.765 & $(1.067,2.892)$ & \\
\hline ENE & & & $0.005^{*}$ \\
\hline Negative & 1 & & \\
\hline Positive & 1.86 & $(1.202,2.878)$ & \\
\hline
\end{tabular}

2 Abbreviations: *, statistically significant $(\mathrm{p}<0.05)$; ENE, extranodal extension;

3 LMR, lymphocyte to monocyte ratio. 
Table 6(on next page)

Different studies about PNI in HNSCC. 
1 Table 6. Different studies about PNI in HNSCC.

\begin{tabular}{|c|c|c|c|c|c|}
\hline Reference & Site & $\begin{array}{l}\text { Case } \\
\text { number }\end{array}$ & $\begin{array}{l}\text { Cut off for } \\
\text { PNI }\end{array}$ & $\begin{array}{l}\text { Primary treatment } \\
\text { strategy }\end{array}$ & $\begin{array}{l}\text { Statically significant } \\
\text { Outcome measurement }\end{array}$ \\
\hline Bruixola $G$ et al. ${ }^{15}$ & $\begin{array}{l}\text { Locoregionally advanced } \\
\text { HNSCC }\end{array}$ & 145 & 45 & $\begin{array}{l}\text { ICT followed by } \\
\text { CCRT }\end{array}$ & OS \\
\hline Kono et al. ${ }^{26}$ & HNSCC & 101 & 40 & Radiotherapy & toxicity of radiotherapy \\
\hline Chang et al. ${ }^{27}$ & $\begin{array}{l}\text { Advanced oral cavity, } \\
\text { oropharynx, hypopharyngeal } \\
\text { cancer }\end{array}$ & 143 & 36 & CCRT & $\begin{array}{l}\text { treatment tolerance and } \\
\text { toxicity of CCRT }\end{array}$ \\
\hline Fu et al. ${ }^{28}$ & $\begin{array}{l}\text { Laryngeal squamous cell } \\
\text { carcinoma }\end{array}$ & 975 & 48.65 & Radical surgery & DSS and OS \\
\hline Our current study & $\begin{array}{l}\text { Advanced stage p16 negative } \\
\text { OPSCC }\end{array}$ & 142 & 50.5 & CCRT & OS \\
\hline
\end{tabular}

2 Abbreviations: PNI, prognostic nutritional index; HNSCC, head and neck squamous cell carcinoma; OPSCC,

3 oropharyngeal squamous cell carcinoma; ICT, induction chemotherapy; CCRT, concurrent chemoradiotherapy; OS,

4 overall survival; DSS, disease-specific survival. 
Table 7 (on next page)

Different studies about LMR in HNSCC. 
1 Table 7. Different studies about LMR in HNSCC.

\begin{tabular}{|c|c|c|c|c|c|}
\hline Reference & Site & $\begin{array}{l}\text { Case } \\
\text { number }\end{array}$ & $\begin{array}{l}\text { Cutoff for } \\
\text { LMR }\end{array}$ & $\begin{array}{l}\text { Primary treatment } \\
\text { strategy }\end{array}$ & $\begin{array}{l}\text { Statically significant } \\
\text { Outcome measurement }\end{array}$ \\
\hline Takahashi et al. ${ }^{11}$ & $\begin{array}{l}\text { Oropharyngeal } \\
\text { carcinoma }\end{array}$ & 75 & 4.97 & \multicolumn{2}{|c|}{$\begin{array}{l}\text { Heterogeneity ( } 76 \% \text { of OS } \\
\text { population were CRT) }\end{array}$} \\
\hline Tham et al. ${ }^{30}$ & HNSCC & 123 & 2.8 & Radical surgery & Event free survival \\
\hline Furukawa et al. ${ }^{31}$ & Tongue cancer & 103 & 4.29 & Radical surgery & OS \\
\hline Yang et al. ${ }^{32}$ & $\begin{array}{l}\text { Hypopharyngeal } \\
\text { carcinoma }\end{array}$ & 197 & 2.98 & Not well documented & OS, DSS and DFS \\
\hline Kano et al. ${ }^{33}$ & $\begin{array}{l}\text { Oropharyngeal, } \\
\text { hypopharyngeal, and } \\
\text { laryngeal cancers }\end{array}$ & 285 & 3.22 & Concurrent CRT & OS and DFS \\
\hline Our current study & $\begin{array}{l}\text { Advanced stage p16 } \\
\text { negative OPSCC }\end{array}$ & 142 & 4.45 & Concurrent CRT & OS, DSS and DFS \\
\hline
\end{tabular}

2 Abbreviations: LMR, lymphocyte to monocyte ratio; HNSCC, head and neck squamous cell carcinoma; OPSCC,

3 oropharyngeal squamous cell carcinoma; CRT, chemoradiotherapy; OS, overall survival; DSS, disease-specific

4 survival; DFS, disease-free survival.

5 
Figure 1

Receiver operating characteristic curves

Receiver operating characteristic curves for predicting the survival outcome. (A) pretreatment prognostic nutritional index (PNI). (B) pretreatment lymphocyte to monocyte ratio (LMR).
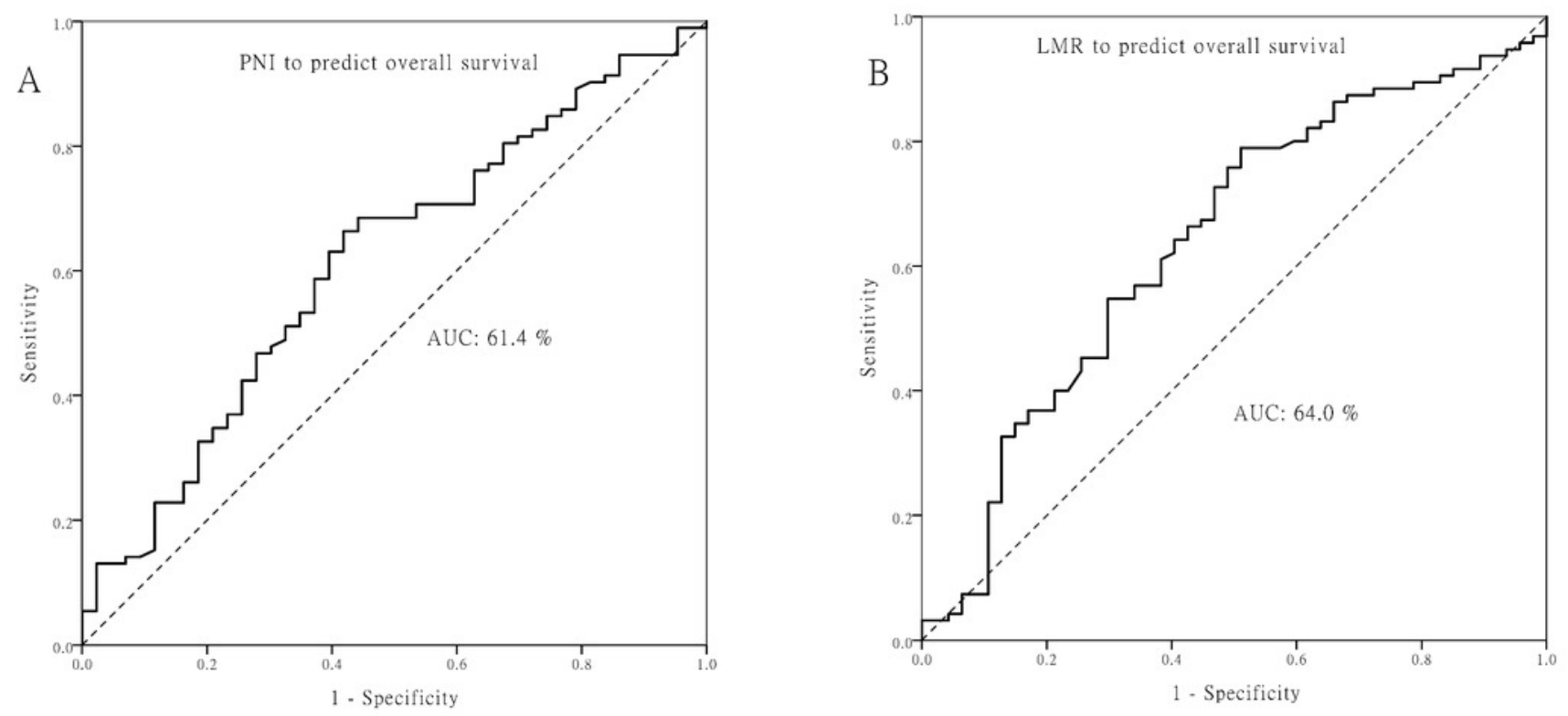
Figure 2

\section{Kaplan-Meier survival curves}

Kaplan-Meier survival curves by different level of pretreatment prognostic nutritional index (PNI). (A) overall survival. (B) disease-specific survival. (C) disease-free survival.
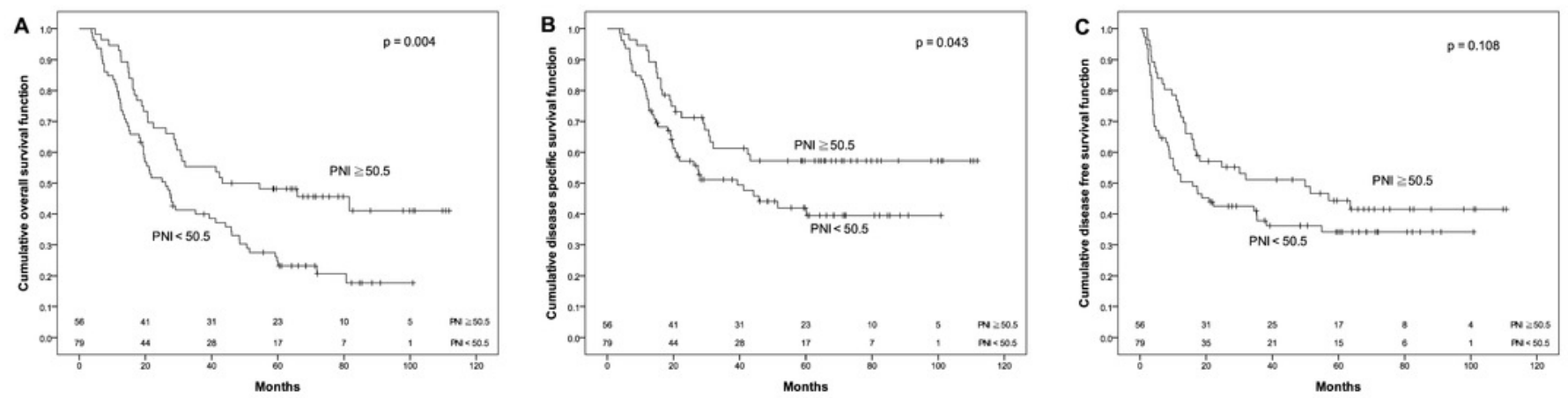
Figure 3

\section{Kaplan-Meier survival curves}

Kaplan-Meier survival curves by different level of pretreatment lymphocyte to monocyte ratio (LMR). (A) overall survival. (B) disease-specific survival. (C) disease-free survival.
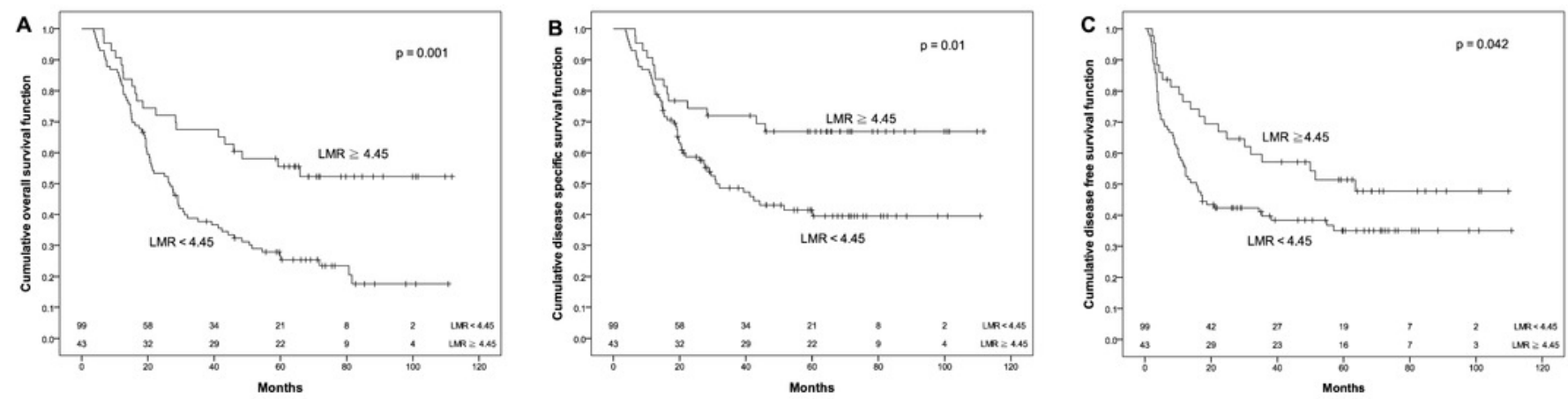